\title{
Feature
}

\section{From the National Academy of Sciences}

\section{Glycoscience: Integrating a Key Macromolecule More Fully into the Curriculum}

\author{
Katherine Bowman and Douglas Friedman
}

Board on Life Sciences and Board on Chemical Sciences and Technology, National Research Council, Washington, DC 20001

\section{WHAT IS GLYCOSCIENCE AND WHY IS IT IMPORTANT?}

Glycans ${ }^{1}$ form one of the four basic classes of macromolecules in living systems, along with nucleic acids, proteins, and lipids. They are composed of individual sugar units that can be linked to one another in multiple ways, enabling them to form complex three-dimensional structures. All living cells are coated on their cell membranes with glycans or include glycan polymers in their cell walls. Glycans are also attached to specific locations on many cellular proteins and are important components of the extracellular matrix. Life as we know it is not possible without glycans.

Not surprisingly for such a large and diverse class of molecules, glycans play numerous roles in living systems. Because of their ubiquity on cell surfaces, they are key components of biological interfaces, including those found between two cells, between a cell and its extracellular environment, or between a host cell and a microorganism. As a result of their presence at such interfaces, glycans are involved in molecular recognition and signaling processes. They are also important molecules in cell adhesion and cell movement, for example, in the migration of cells to specific tissue locations during

DOI: $10.1187 /$ cbe.12-12-0217

Address correspondence to: Katherine Bowman (kbowman@nas .edu) and Douglas Friedman (dfriedman@nas.edu).

${ }^{1}$ As used in the National Research Council report, glycan refers to "any sugar or assembly of sugars, in free form or attached to another molecule" (such as in a glycoprotein or a glycolipid) (NRC, 2012b, p. 188). The term is used interchangeably with terms such as carbohydrate and saccharide to help emphasize a unified vision for the study of glycoscience.

(C) 2013 K. Bowman and D. Friedman. CBE-Life Sciences Education (C) 2013 The American Society for Cell Biology. This article is distributed by The American Society for Cell Biology under license from the author(s). It is available to the public under an AttributionNoncommercial-Share Alike 3.0 Unported Creative Commons License (http:/ / creativecommons.org/licenses/by-nc-sa/3.0).

"ASCB ${ }^{\mathbb{R} \text { " }}$ and "The American Society for Cell Biology ${ }^{\mathbb{R} \text { " }}$ are registered trademarks of The American Society for Cell Biology. development or the trafficking of white blood cells to sites of injury. Meanwhile, glycans on proteins inside cells participate in cells' responses to incoming biological signals, for example, helping to modulate gene expression and protein functions.

Glycan polymers such as cellulose are important components of plant cell walls. Understanding how such walls are assembled, and how they can be deconstructed, is fundamental to basic plant biology but also in the development of applications such as efficient conversion of biomass into fuels. Glycan polymers derived from plants and other organisms can also serve as sources of new materials with wide-ranging applications from tissue-engineering scaffolds to flexible electronic displays.

\section{TRANSFORMING GLYCOSCIENCE}

Achieving an understanding of the structures and functions of glycans is fundamental to understanding biology. A recent report from the National Research Council, Transforming Glycoscience: A Roadmap for the Future (NRC, 2012b; Figure 1), explores this important area of the life sciences and provides recommendations for bringing glycoscience into research and teaching, such that it will become a widely recognized and integrated discipline, rather than a niche area studied by a small number of specialists.

Unlike DNA and proteins, glycans are not generated by following a template. Instead, the reactions that link individual sugar units are influenced by factors that include cellular metabolism, cell type, developmental stage, and nutrient availability. These factors provide opportunities for substantial diversity but at the same time make glycans more difficult to study and manipulate in the laboratory. In comparison, genetic research has undergone an explosion over the past 25 years. Almost any scientist can now draw on a wide array of tools and techniques that enable him or her to incorporate the study of nucleic acids into research and into hands-on laboratory experiences for students.

In its report, the NRC committee made five recommendations for how to advance and transform the field of 


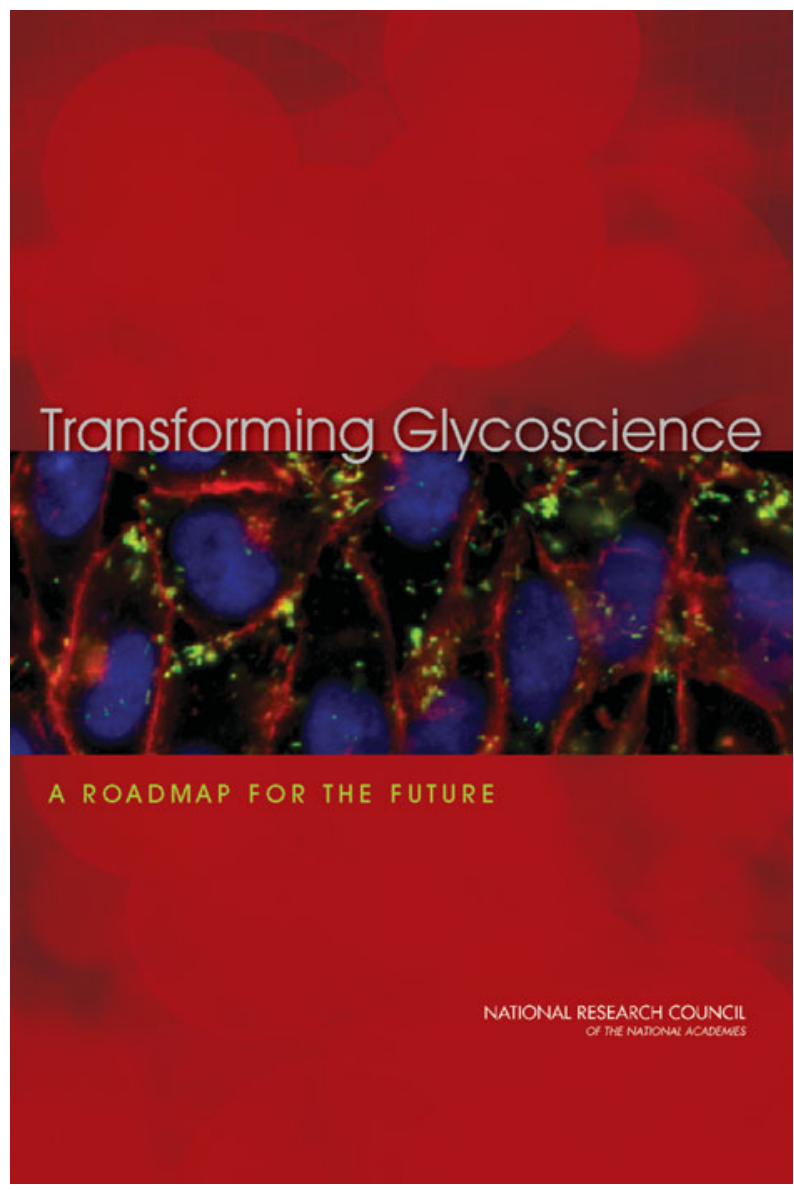

Figure 1. Cover of 2012 NRC Report on Glycoscience (NRC, 2012b).

glycoscience; these were largely focused on a tool kit that would enable glycoscience research to be conducted. Although there is a diverse set of tools for studying glycans, and progress continues to be made in improving this tool kit, many gaps remain. Many of the techniques commonly needed (e.g., methods for glycan synthesis and analysis) still require specialized equipment or expertise. These factors continue to limit the field and keep it from being widely integrated into the research of all scientists who might have an interest in addressing aspects of glycans in their work or their teaching.

In addition to its set of technical recommendations, the committee drew the following conclusion, which emphasizes the role of education as an underpinning foundation for the future of the field:

The committee concludes that integrating glycoscience into relevant disciplines in high school, undergraduate, and graduate education, and developing curricula and standardized testing for science competency would increase public as well as professional awareness. (NRC, 2012b, p. 12)

A key aspect of the challenge to transform glycoscience will be educating the next generation of scientists on what glycans do and how they can be used to help address needs in areas such as health, energy, and materials science. The central functions of DNA, RNA, and proteins are already broadly discussed in high school and undergraduate courses, but glycans generally remain understudied. Examples drawn from glycoscience may be suitable additions to many areas of the science curriculum, and their inclusion will help ensure that students have an understanding of the diverse functions played by this key class of macromolecules.

\section{GLYCOSCIENCE IN AN INTEGRATIVE BIOLOGY CURRICULUM}

Participation by the education community is needed to develop a wider appreciation of the importance of glycans. Examples of key roles played by glycans, discussion of stillunanswered questions about their structures and functions, and information on how they can be manipulated or applied to help tackle practical problems are topics that could be meaningfully introduced in a variety of places in an undergraduate or graduate science curriculum.

A report released by the National Research Council nearly a decade ago, BIO2010: Transforming Undergraduate Education for Future Research Biologists (NRC, 2003), provides a starting point for thinking about how glycoscience-based examples can be effectively incorporated into classes. A central theme of the BIO2010 report was the need to structure undergraduate biology education to reflect the integrated and interdisciplinary contributions from the chemical, mathematical, physical, computational, engineering, social, and behavioral sciences. The report includes several case studies that help illustrate how some of these connections can be drawn. Case study 3 discusses the role of glycans in biological recognition in the context of influenza:

In his organic chemistry course Jerry Mohrig integrates
material on carbohydrates by having a capstone to his
yearlong course. This capstone is called "Why do we
get the flu every year?"... Although the basics of car-
bohydrate and amino acid chemistry are taught as part
of most second-term organic chemistry courses, many
students would be hard pressed to recognize or ap-
preciate the great importance that carbohydrates have
in biochemical recognition... . Dr. Mohrig believes that
it is not enough to teach future biologists the organic
chemistry of small molecules if they never see how
this knowledge can be applied to biological molecules
of consequence. It is important that students see that
they can make sense of how to relate complex organic
molecules to biological questions and develop the con-
fidence to do so. Since he has been teaching the flu
module, he has seen a significant increase in the inter-
est in organic chemistry of the many biology students
in the course.... Student opinion on the value of the
module increased if they later took a biology course in
which the professor discussed the chemistry of carbo-
hydrates. The biology faculty had to communicate to
their students that chemistry was essential to fully un-
derstand the biological system. For more information:
http:/ / mc2.cchem.berkeley.edu/modules / flu. (NRC,
2003, pp. $64-65$ )

This provides just one example of how a module that discusses the roles of glycans in a context students can relate to (everyone gets the flu) can foster an increased appreciation for what glycans do, as well as help to connect the subjects students learn in their chemistry and biology classes. A few other possible ideas, drawn from examples in the NRC 
glycoscience report, are provided below. These represent just the tip of the iceberg; many others could be developed.

\section{Beyond Metabolism: A Few Examples of How Glycoscience Could Be Brought into Curricula}

Understanding Complex Systems: The Flow of Biological Information and the Roles of Glycans in This Process. A central tenet of biology is that cellular DNA encodes the genetic make-up of an organism; the message is carried farther by being transcribed into RNA, which is translated into proteins that carry out many of a cell's functions. Multiple proteins interact in signaling cascades, multiple cells and cell types are structured into tissue and organ systems within an individual, and multiple individual organisms interact with one another and with their environments in communities, all of which evolve and change over time. It is certainly recognized that the situation is more nuanced than this simplified picture portrays. But an appreciation of the complexity of living systems and a basic understanding of how genetic information is expressed and modified to produce an outward phenotype are core concepts in the life sciences. This basic competency, for example, was included in the reports Vision and Change in Undergraduate Biology Education: A Call to Action (AAAS, 2011) and Scientific Foundations for Future Physicians (Association of American Medical Colleges [AAMC] and Howard Hughes Medical Institute, 2009); these reports helped inform and influence the recent revision of the Medical College Admission Tests (AAMC, 2012).

Glycans fit well into a discussion of information flow in complex living systems because they provide a level of diversity that extends beyond what is encoded in the genome and thus have the potential to significantly increase the available information content. Glycosylation is not a passive posttranslational modification; glycans frequently modulate or regulate protein functions. The significance of glycosylation can be seen in the fact that appropriate glycosylation of many therapeutic proteins affects their circulation time in blood and their biological activity. Aberrant glycosylation is a hallmark of cancer, and patterns of such alterations can be used as biomarkers to identify types of disease. A discussion of glycans and their effects can and should be incorporated into discussions of the information provided by the key molecules that make up living systems and how they interact with each other to ultimately make us who we are.

Glycans and Infectious Disease: An Opportunity to Bring Together Biological, Pharmaceutical, Epidemiological, and Social Sciences. Glycans play key roles in the interactions of microorganisms with host cells that extend far beyond the influenza example mentioned above. A discussion that includes host-pathogen interactions, immune responses and pathogen strategies for immune evasion, efforts to develop effective vaccines and therapeutics, and other biological questions also provides an opportunity to incorporate aspects of the social and behavioral sciences. The intersection of the life sciences with these fields is a message that has been emphasized in reports such as BIO2010, Vision and Change, and others.

An example, such as HIV, can help illustrate some of these concepts. One of the key molecules that form the HIV envelope is gp120, which binds to the CD4 receptor on the surface of target cells and helps initiate the process of viral entry. Gp120 is heavily glycosylated, and its glycosylation changes over the course of infection. In this way, glycans on gp120 help to form a "glycan shield" that blocks antibodies from being able to bind and neutralize the virus. On the other hand, the immune system can develop broadly neutralizing antibodies that prevent HIV from infecting new cells, and many of these recognize glycan epitopes on the HIV surface. As a result, the glycans on HIV are important in enabling it to carry out its functions, while also forming part of a coevolving system between the virus and the human host.

A range of complementary topics drawing on the social and behavioral sciences could accompany a fuller discussion of the infection and response process, including the ethics of clinical research trials, social perceptions of disease and how those affected by a particular disease are perceived, the epidemiology and modeling of disease burdens in different subpopulations, or factors that affect disease risk or treatment. Because many vaccines target carbohydrate antigens, and many drugs are proteins whose correct glycosylation is important for biological activity, a discussion of glycans in the context of disease also provides a link to applied science, such as strategies for the development of new therapeutics and the process of pharmaceutical production. Such discussions can introduce concepts, highlight connections and interactions between the life sciences and other fields, and provide real-world examples to help students relate to the material.

Beyond Health: Roles for Glycans in Energy and Materials Science. The contributions biology can make to helping address societal challenges in areas such as agriculture and energy have also been highlighted in previous NRC reports, including A New Biology for the 21st Century (NRC, 2009), Research at the Intersection of the Physical and Life Sciences (NRC, 2010), and Sustainable Development of Algal Biofuels (NRC, 2012a). Student interest in alternative forms of energy represents one opportunity to bring a glycoscience-based example into the curriculum, since an improved understanding and application of glycoscience knowledge can contribute to "green" goals.

Glycan polymers, such as cellulose and hemi-cellulose, are integral components of cell walls. To economically break down the cellulosic biomass in plants and convert it into liquid biofuels, you must first overcome the phenomenon of recalcitrance-resistance of plant cell walls to degradation. Students might learn not only about the basic structures of plant cell walls, but also that much remains to be understood about how these walls are formed, how the molecules within them interact, and how they can be modified in useful ways. For example, crops might be genetically modified to maximize biomass production and improve conversion rates, while minimizing inputs of fertilizer and water. Knowledge derived from glycoscience and from an improved understanding of cell wall biology might also be applied to develop new enzymes and other catalysts that can improve the conversion and fermentation process. In addition to potential contributions to biofuels, glycan polymers, such as cellulose and its derivatives, can also be used in new materials science applications. For example, nanocrystals extracted from highly structured regions of cellulose can be used to develop composite materials and replacements for 
petroleum-based plastics. Such discussions provide an opportunity to highlight how glycans fit into the life sciences more broadly than in health and disease and may prove interesting to students, because of their links to practical applications in emerging research areas.

\section{CONCLUSION}

The examples above represent only a few ideas for how educators might incorporate glycoscience into their lesson plans. As the NRC report emphasizes,

\begin{abstract}
Glycoscience is a vibrant field filled with challenging problems. It can make contributions toward understanding and improving human health, creating nextgeneration fuels and materials, and contributing to economic innovation and development. Now is the time for glycoscience to be embraced broadly by the research community. Drawing in members from the full spectrum of chemistry, biology, materials science, engineering, medicine, and other disciplines will be needed to address the technical challenges described here. Although these challenges are substantial and complex, the results of achieving these goals have the potential to impact science in exciting ways. (NRC, 2012b, p.12)
\end{abstract}

Students will not only be the future researchers and innovators, but also need to be informed citizens. Assuring that they receive a grounding in the chemistry, biology, and functions of glycans along with the standard instruction in the areas of nucleic acids, proteins, lipids, and other core components of the cell in the life and chemical sciences curricula can provide a foundation to help transform the glycoscience field and achieve the vision of a stronger curriculum. Realizing this aim of the report will provide benefits both for the scientific understanding of living systems and for societal goals in the effective manipulation and utilization of biosystems.

\section{The following individuals were members of the Committee on Assessing the Importance and Impact of Glycomics and Glycosciences:}

David Walt (Chair), Tufts University

Kiyoko F. Aoki-Kinoshita, Soka University, Japan

Brad Bendiak, University of Colorado, Denver

Carolyn R. Bertozzi, University of California, Berkeley

Geert-Jan Boons, University of Georgia
Alan Darvill, University of Georgia

Gerald Hart, Johns Hopkins University

Laura L. Kiessling, University of Wisconsin, Madison

John Lowe, Genentech, Inc.

Robert Moon, Forest Products Laboratory, U.S. Forest Service

James C. Paulson The Scripps Research Institute

Ram Sasisekharan, Massachusetts Institute of Technology

Ajit P. Varki, University of California, San Diego

Chi-Huey Wong, Academia Sinica, Taiwan, and The Scripps

Research Institute

\section{ACKNOWLEDGMENTS}

We particularly thank Ajit Varki, Distinguished Professor of Medicine and Cellular and Molecular Medicine and Co-Director of the Glycobiology Research and Training Center, University of California, San Diego, for comments and suggestions on the manuscript. The report (NRC, 2012b) was supported by the National Institutes of Health, the National Science Foundation, the U.S. Department of Energy, the Food and Drug Administration, and the Howard Hughes Medical Institute.

\section{REFERENCES}

American Association for the Advancement of Science (2011). Vision and Change in Undergraduate Biology Education: A Call to Action, Washington, DC.

Association of American Medical Colleges (AAMC) and Howard Hughes Medical Institute (2009). Scientific Foundations for Future Physicians. Report of the AAMC-HHMI Committee, Washington, DC: AAMC.

AAMC (2012). MCAT 2015: A Better Test for Tomorrow's Doctors. Preview Guide for the MCAT 2015 Exam (2nd ed), Washington, DC.

National Research Council (NRC) (2003). BIO2010: Transforming Undergraduate Education for Future Research Biologists, Washington, DC: National Academies Press.

NRC (2009). A New Biology for the 21st Century, Washington, DC: National Academies Press.

NRC (2010). Research at the Intersection of the Physical and Life Sciences, Washington, DC: National Academies Press.

NRC (2012a). Sustainable Development of Algal Biofuels, Washington, DC: National Academies Press.

NRC (2012b). Transforming Glycoscience: A Roadmap for the Future, Washington, DC: National Academies Press. 